\title{
Technical Report
}

\section{Personnel:}

\section{Faculty}

PI: E. Kent Barefield, 12 month, $10 \%$ time, no cost to project.

co-PI: Charles L. Liotta, 12 month, 5\% time, no cost to project.

co-PI: Hem-y M. Neumann, 12 month, $10 \%$ time, no cost to project.

\section{Post-Doctorals}

Dr. Vikram Kumar, Ph.D., June 1997, Univ. of Georgia: 1 mon (100\% time.)

\section{Graduate Students}

Mr. Anthony Belcher, first year graduate student: 9 mon, $40 \%$ time (full-time graduate student)

\section{Undergraduate Students}

Ms. Amanda Chalfant, junior special problems (undergraduate research) student: one quarter $(2 \mathrm{~h}$ credit, $\geq 6 \mathrm{~h} / \mathrm{wk})$

\section{Research Progress:}

Work during the past year has been concentrated in three areas: Analysis of the Relative Contributions of Thermal versus Radiolytic Pathways for Complexant Decomposition in Tank 101SY. Synthesis of Potential Precursors to $\mathrm{HNO} / \mathrm{NO}^{-}$, and Analysis of the Kinetics of Decomposition of Piloty's Acid at High $\left[\mathrm{OH}^{-}\right]$. The undergraduate student worked on the aluminum catalyzed reactions of nitrite ion with 2-hydroxyethylamines. This is a follow-up to earlier work done under Westinghouse Hanford and PNNL funding that will be expanded to include an exploration of the complexation of nitrite ion by aluminum when Ms. Chalfant's lab skills are sufficiently established. A brief synopsis of work in each of the first three areas follows.

Analysis of the Relative Contributions of Thermal versus Radiolytic Pathways for Complexant Decomposition in Tank 101-SY. We have attempted to utilize the experimentally determined rate constants for thermal and $G$ values for radiolytic decomposition of HEDTA and glycolate in simulated waste mixtures and the tank fill data for tank 101-SY, along with its physical parameters collected by Agnew,' to 1) determine the ratio of thermal to radiolytic decomposition, and 2) the expected composition in the tank today. The rate constants utilized were those determined by us at Georgia Tech in earlier work supported by DOE through contracts with Westinghouse Hanford and PNNL. ${ }^{2} \mathrm{G}$ values were taken from work by Meisel $^{3}$ at ANL and by Camaioni at PNNL. A large number of assumptions must be made in this analysis, but the results suggest that in the original (1978) tank mixture about $15 \%$ of HEDTA reactions and $95 \%$ of glycolate decompositions occur by thermal pathways. The predicted low concentration of HEDTA that should have been present in 1994 is consistent with analyses of actual tank waste by Campbell and co-workers ${ }^{5}$ at PNNL, (report PNNL11307/UC-601, September 1996). A publication describing this analysis is in preparation for 
submission to J. N ucl. Tech.

Synthesis of Potential Precursors to $\mathrm{HNO}_{\mathrm{NO}}^{-}$. As outlined in our proposal a major focus of our current DOE-funded research is to investigate the properties and reactivity of NO- as a function of its spin-state (singlet or triplet). This anion is believed to be an intermediate in the reaction of nitrite ion with metal complexants present in certain Hanford HLW and the partitioning of NO- between the various nitrogen products may depend upon spin s\&ate. Both the ground state of the ion in condensed phase and life-time of the excited state are currently unknown. To study the properties and reactivity of NO- will require the development of new methods of generation of this short-lived precursor. We expect to utilize the known DielsAlder adduct of $\mathrm{HNO}$ and $9,10-$ dimethylanthracene (DMA, I), II, and derivatives as the basis for this portion of our work.

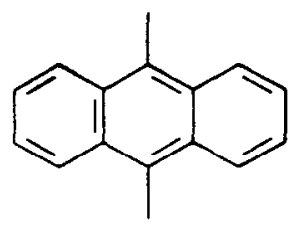

I

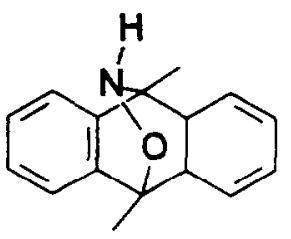

II However, the projected requirements for the quantity of I required coupled with its high cost $(\$ 54 / \mathrm{g})$, and our limited materials budget necessitated the development of a cost-effective synthesis, preferably one that can be easily extended to derivatives. Tony Belcher has succeeded in adapting the well-known transition metal catalyzed coupling of aryl halides with

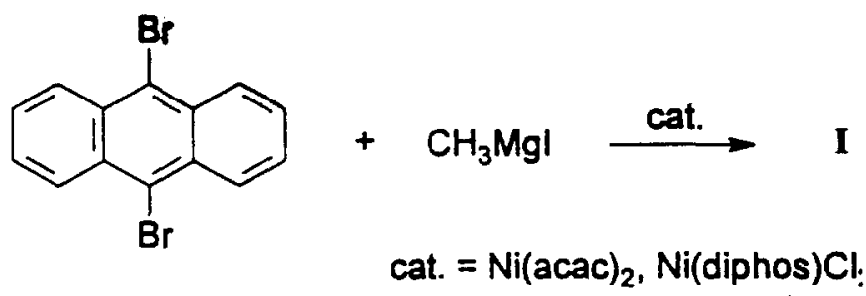

Grignard reagents to provide DMA from 9, 10-dibromoanthracene (\%2/g) and $\mathrm{CH}_{3} \mathrm{MgBr}$ in ca. $80 \%$ yield on at least a $10 \mathrm{~g}$ scale. This is more than adequate for our purposes. This reaction has been extended to include $\mathrm{EtMgBr}$ (75\% yield) and preliminarily to $\mathrm{i}-\mathrm{PrMgBr}$. The product of the latter has not yet been purified nor have reaction parameters been optimized. This method is superior to the method reported by Czarnick (lithium-bromine exchange in 9,10-dibromoanthracene with butyl lithium followed by reaction with alkyl halide) in both ease of manipulations and yield. If the i-propyl derivative is realized in decent yield then the method will be superior in scope as Czarnick failed to prepare derivatives with sterically hindered alkyl halides having $\boldsymbol{\beta}$-hydrogens. Publication of the synthetic methodology as a note in J. $\mathbf{O ~ r g}$. Chem. is anticipated.

The literature synthesis of II has been successfully completed, although the product is apparently much more thermally, or photochemically, sensitive than suggested by the literature. Exploration of the thermal and photochemical reactivities of II are underway.

Analysis of the Kinetics of Decmposition of Piloty's Acid at High [O $\mathrm{H}^{-}$]. It is generally agreed that Piloty's acid $\left(\mathrm{PhSO}_{2} \mathrm{NHOH}\right)$ decomposes under basic conditions to produce NO-. The kinetics of this reaction have been studied several times, most recently by Bonner, as a function of $\mathrm{pH}$. However, none of these studies have been done at base concentrations comparable to those in tank wastes. We have initiated a study of this reaction for $\left[\mathrm{OH}^{-}\right]$ 
from 0.5 to $2.5 \mathrm{M}$ using ${ }^{1} \mathrm{H}$ NMR for measurement of the decay of the acid (initially $0.05 \mathrm{M}$ ). Our data suggest that the reaction of the acid decreases at the higher [OH-] concentrations. This is consistent with the formation of doubly deprotonated acid, which is much less reactive than the monodeprotonated form. A second ionization constant can be extracted from the data. However, whereas we have data that are internally consistent, our rate constants are smaller than those of Bonner. There are some differences in conditions (atmosphere, ionic strength) that must be explored to determine what is causing the difference in rate. As part of this study we have have found that there is no effect on the kinetics of decomposition upon addition of the known NO- trapping agent $\mathrm{Ni}(\mathrm{CN})_{4}^{2-}$.

The possibility of reversal of the reaction $\mathrm{PhSO}_{2} \mathrm{NHO}^{-}=\mathrm{PhSO}_{2}^{-}+\mathrm{HNO}$ was suggested by Bonner, although the data were not unambiguous. For this reaction to be reversible, the rate of the reverse reaction must be competitive with the rate of deprotonation of HNO. Our current expectations for the reactivity of HNO would suggest that this would not be the case. However, it would possibly not be unreasonable for HON. The assignment of structure to the major monodeprotonated species was based upon nuclear Overhauser enhancement factors. Even if this assignment is correct, it does not exclude the presence of some of the Ndeprotonated form, nor its more rapid decomposition. Since the rate of decomposition is much slower than the NMR time scale, equilibration between $\mathrm{O}$ - and $\mathrm{N}$-deprotonated forms is not an issue.

\section{References}

(1) "Hanford Tank Chemical and Radionuclide Inventories: HDW Model Rev. 4," Agnew S.F., et al. LA-UR-96-3860, January 1997.

"Mechanisms of Gas Generation from Simulated SY Tank Farm Wastes: FY 1995

Progress Report," Barefield, E.K.; Boatright, D.; Desphande, A.; Doctorovich, F.; Liotta, C.L.; Neumann, H.M.; Seymore, S. PNNL-11247, 1996.

"Mechanisms of Gas Generation from Simulated SY Tank Farm Wastes: FY 1994 Progress Report," E.K. Barefield, D. Boatright, A. Desphande, F. Doctorovich, C.L. Liotta, H.M. Neumann, and S. Seymore, PNL-10822, 1995.

"Synthetic Waste Chemical Mechanism Studies," E.C. Ashby, A. Annis, E.K. Barefield, D. Boatright, F. Doctorovich, C.L. Liotta, H.M. Neumann, A. Konda, C.F. Yao, K. Zhang, and N.G. McDuffie, WHC-EP-0823, 1994.

"Radiolytic and Radiolytically Induced Generation of Gases from Synthetic Wastes," Meisel, D., et al., ANL-93143, October 1993.

“Organic Tanks Safety Program FY96 Waste Aging Studies," Camaioni, D.M., et al., PNNL-11312/UC-2030, October 1996.

"Flammable Gas Safety Program; Actual Waste organic Analysis TY 1996 Program Report," Campbell, J.A., et al., PNNL- 11307/UC-601, September 1996. 